Check for updates

Cite this: Chem. Sci., 2019, 10, 10388

๑ All publication charges for this article have been paid for by the Royal Society of Chemistry

Received 17th June 2019

Accepted 19th September 2019

DOI: $10.1039 / \mathrm{c} 9 \mathrm{sc0} 02961 \mathrm{c}$

rsc.li/chemical-science

\section{Bottom-up self-assembly of heterotrimeric nanoparticles and their secondary Janus generations $\uparrow$}

\author{
Jianye $\mathrm{Fu}{ }^{\mathrm{a}}$ Zhengying Gu, ${ }^{\mathrm{a}}$ Yang Liu, ${ }^{\mathrm{a}}$ Jun Zhang, ${ }^{\mathrm{a}}$ Hao Song, ${ }^{\mathrm{a}}$ Yannan Yang, ${ }^{\mathrm{a}}$ \\ Yang Yang, ${ }^{a}$ Owen Noonan, ${ }^{a}$ Jie Tang ${ }^{* a}$ and Chengzhong Yu iD *ab
}

A bottom-up self-assembly approach is developed for the synthesis of ABC type heterotrimeric nanoparticles, which can be converted into secondary Janus-type silica derivatives. Compared to spherical ones, Janus silica nanoparticles stimulate stronger phagocytosis and transcytosis through intestinal epithelial microfold cells and exhibit higher cargo transport across an in vitro epithelial monolayer model mimicking the human intestinal epithelium.

\section{Introduction}

Janus objects, named after the double-faced Roman god, ${ }^{\mathbf{1}}$ are fascinating in nature. ${ }^{2}$ One example is a bacteriophage (i.e., a virus that infects bacteria), which shows Janus shapes and extraordinary properties. ${ }^{3}$ The past ten years have witnessed vigorous progress in the study of Janus nanoparticles especially the ones with morphological asymmetry and how the asymmetric nanoparticles adjust their functions. ${ }^{\mathbf{1 4 - 6}}$ Nanoparticles with morphological asymmetry, e.g. , heterodimeric AB type with two domains and heterotrimeric $\mathbf{A B C}$ type with three different domains in one particle, can be fabricated generally through top-down or seed-mediated approaches. ${ }^{7-11}$ However, using molecular assembly to synthesize Janus nanoparticles is extremely difficult, and to date there is no report on the preparation of $\mathbf{A B C}$ type heterotrimeric nanoparticles through a onestep bottom-up assembly approach.

Janus nanoparticles with various structures and compositions have wide-spread applications in catalysts, ${ }^{12}$ nano/micromotors ${ }^{13,14}$ and nanomedicine..$^{15-17}$ The interaction between nanoparticles and cells plays a central role in cellular delivery applications. ${ }^{18-20}$ The impact of the size, shape, composition, aspect ratio and orientation of nanoparticles interacting with cells has been extensively reported..$^{\mathbf{3 2 1}-23}$ Nevertheless, there are few reports where the difference between Janus and spherical nanoparticles is directly compared.

${ }^{a}$ Australian Institute for Bioengineering and Nanotechnology, The University of Queensland, St Lucia, Brisbane, QLD 4072, Australia.E-mail: j.tang3@uq.edu.au; c. yu@uq.edu.au

${ }^{b}$ School of Chemistry and Molecular Engineering, East China Normal University, Shanghai 200241, P. R. China.E-mail: czyu@chem.ecnu.edu.cn

$\dagger$ Electronic supplementary information (ESI) available. See DOI: $10.1039 / \mathrm{c} 9 \mathrm{sc} 02961 \mathrm{c}$
In the present work, for the first time, we report a bottom-up self-assembly approach of ABC type heterotrimeric nanoparticles (Scheme 1a). The synthesis involves three molecular precursors: 3-aminophenol (AP), formaldehyde (F), and tetraethyl orthosilicate (TEOS) in an ammonia water/ethanol solution containing ethylenediamine (EDA). The hydrolysis and condensation of TEOS firstly results in the formation of silica primary particles (SPPs, 2-3 $\mathrm{nm})^{\mathbf{2 4}}$ and then Stöber spheres with larger particle sizes (several tens of $\mathrm{nm})^{25}$ as building block $\mathbf{A}$. The polymerization of AP and F occurs afterwards, leading to APF oligomers which nucleate as building block B preferentially on one side of block A. Afterwards, SPPs and APF oligomers cocondense, forming building block $\mathbf{C}$ on the other side of $\mathbf{A}$. Eventually, an ABC type heterotrimeric nanoparticle with an acorn-like Janus morphology is constructed, which can be used to generate secondary Janus-type derivatives (Scheme 1b): Janus silica nanoparticles (J-SNPs) by calcination to remove APF. By tuning the reaction conditions, symmetric SNPs (S-SNPs) can

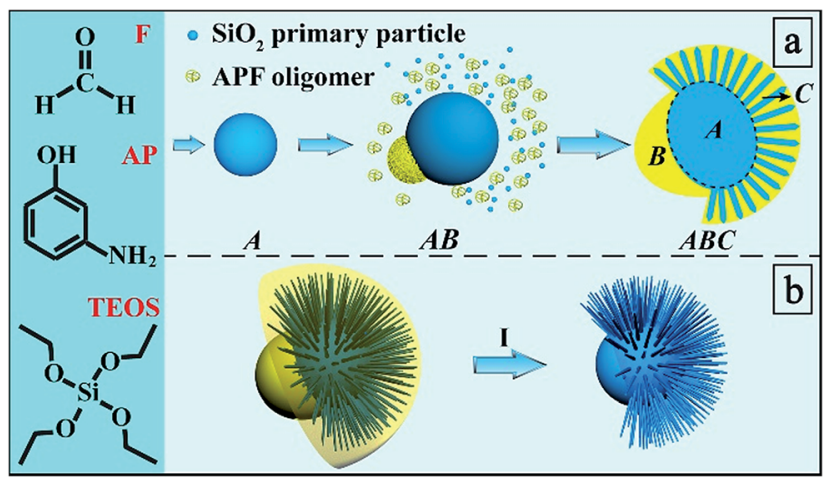

Scheme 1 Synthesis process and mechanism. (a) Bottom-up selfassembly of $A B C$ type heterotrimeric nanoparticles and (b) their conversion into secondary Janus-type silica nanoparticles (I). 
also be prepared. J-SNPs and S-SNPs were well dispersed and stable in PBS solution (Fig. S1 $\dagger$ ), and can be used as a new platform to investigate the impact of symmetry on the interactions between nanoparticles and cells.

\section{Results and discussion}

Transmission electron microscopy (TEM) images of the heterotrimeric nanoparticles (Fig. 1a and b) clearly showed a Janus nanostructure similar to the morphology of the fruit acorn, consistent with scanning electron microscopy (SEM) observations (Fig. 1c). The particles were monodisperse and uniform in size $(\sim 420 \mathrm{~nm})$. As shown in Fig. 1b, the "bulge" (block B) exhibited a hemispherical morphology with a smaller contrast than the "cap" (block C) with spike-like fine nanostructures. Moreover, the core (block A) showed the darkest contrast.
Scanning TEM (STEM) coupled with energy dispersive X-ray spectroscopy (EDS) elemental mapping (C, N, O, and Si) was used to record the heterogeneous composition in the heterotrimeric colloidal nanoparticles. The high-angle annular dark field (HAADF) STEM image (Fig. 1d) showed a similar acorn-like morphology to that in Fig. 1b. According to the corresponding elemental mapping results, the distribution of $\mathrm{C}$ and $\mathrm{N}$ elements exhibited a higher intensity in block $\mathbf{B}$ than in $\mathbf{A}$ and $\mathbf{C}$. The $\mathrm{O}$ and $\mathrm{Si}$ intensity showed an opposite trend, and the $\mathrm{Si}$ signals were hardly observed in block B. The heterogeneous distribution of $\mathrm{C}, \mathrm{N}, \mathrm{O}$, and $\mathrm{Si}$ elements was evident in the overlapped image. The above results indicate that the acorn-like heterotrimeric nanoparticle has a structure as shown in Scheme 1: the "bulge" (block B) is composed of APF without $\mathrm{SiO}_{2}$, the "cap" is a silica-APF composite shell (block $\mathbf{C}$ ), and there is a silica-rich core (block A) inside.
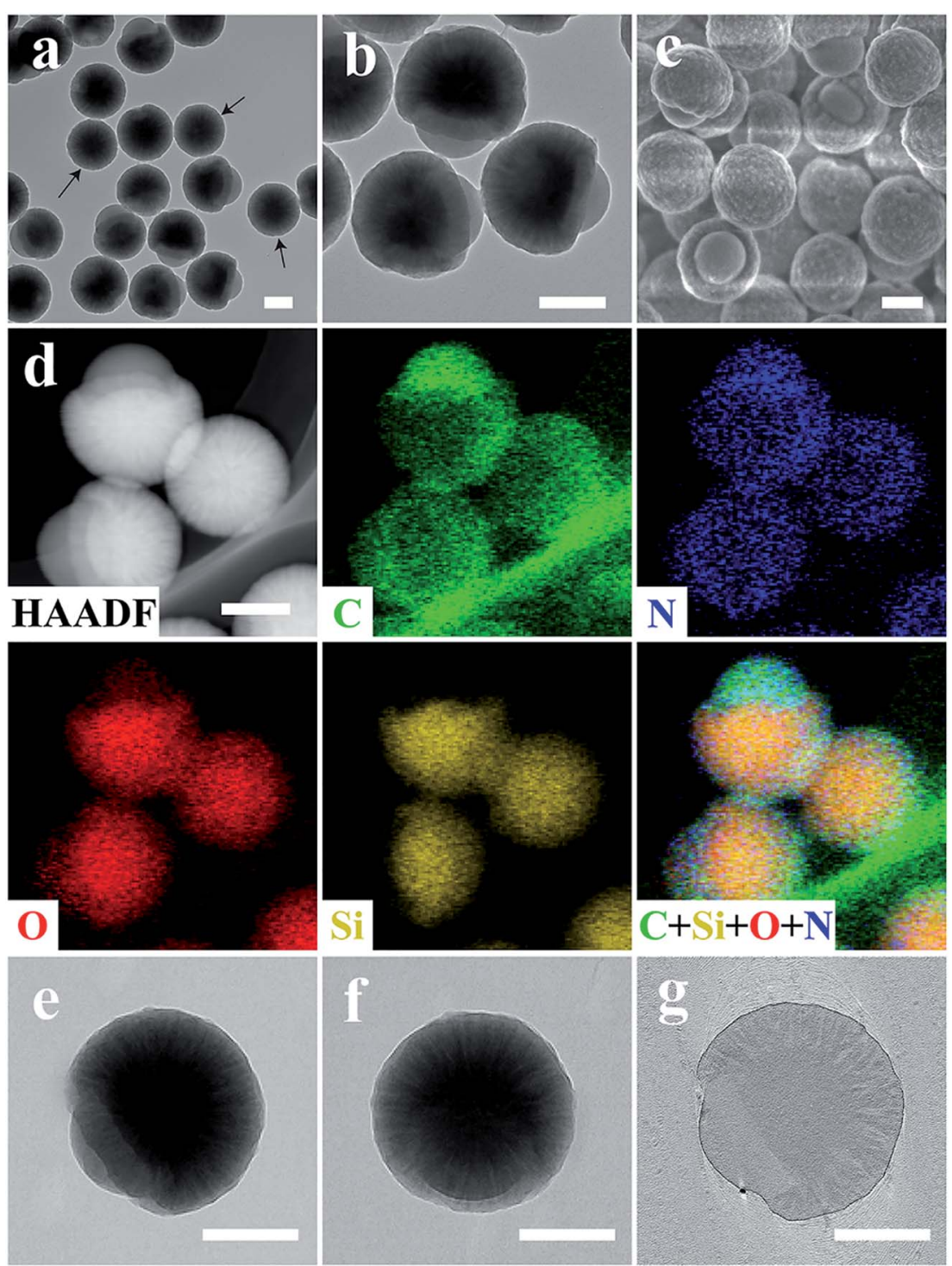

Fig. 1 TEM, SEM, STEM EDS mapping and ET images of the ABC type heterotrimeric nanoparticles. TEM (a and b); SEM (c); HAADF-STEM (d) and the corresponding EDS elemental mapping images and their overlays. Tilting TEM images (e and f, from $+120^{\circ}$ to $0^{\circ}$ ) and the ET slice $(\mathrm{g})$. All scale bars are $200 \mathrm{~nm}$. 
To obtain detailed structural information of Janus nanoparticles, electron tomography (ET) was used..$^{26}$ Tilting the Janus nanoparticles along the $x$-axis resulted in a spherical appearance (Fig. 1e and f), which explains the observation of "spherical" nanoparticles in Fig. 1a (denoted by arrows). An ET slice from the center through the $y-z$ plane of the ABC type heterotrimeric nanoparticle showed clearly three blocks (Fig. 1g).

Thermogravimetric analysis (TGA) was conducted to study the composition ratio in the $\mathbf{A B C}$ type heterotrimeric nanoparticles (Fig. S2 $\dagger$ ). A weight loss of $\sim 40 \%$ was observed, indicating that the weight ratios of the APF polymer and silica are $\sim 40 \%$ and $\sim 60 \%$, respectively. After removing the APF polymer from the ABC type heterotrimeric nanoparticles by calcination at $550{ }^{\circ} \mathrm{C}$, well dispersed J-SNPs were obtained. From the SEM image (Fig. 2a), an AB type heterodimeric Janus morphology was observed with a mean particle size of $370 \mathrm{~nm}$. The Janus structure was confirmed by TEM observation (Fig. 2b). A solid core with a mean size of $230 \mathrm{~nm}$ was observed. The length of the spiky shell was $\sim 70 \mathrm{~nm}$ and the diameter of one spike was around $10 \mathrm{~nm}$. A core with a relatively smooth surface was embedded in a partially open spherical spiky shell as illustrated by the 3D model (Fig. 2c), similar to the moonflower seed pod in nature. Some of the nanoparticles were observed to be fully covered by the silica spikes, which may be because the "dent" part was facing down as supported by the tilting TEM images shown in Fig. 2d and e. Our observations suggest that the APF "bulge" (block B) in the ABC type heterotrimeric nanoparticle was fully removed during calcination, and the core (block A) remained as the solid particle, while the silica-APF composite "cap" (block C) was converted into the spiky silica shell (Fig. 2f).

$\mathrm{N}_{2}$ sorption-desorption analysis of the $\mathbf{A B C}$ type heterotrimeric nanoparticles and J-SNPs is shown in Fig. S3, $\dagger$ demonstrating the mesoporous nature of J-SNPs (Table S1†). The particle sizes of the ABC type heterotrimeric nanoparticles and J-SNPs measured by dynamic light scattering (DLS, Fig. S1†) were slightly larger than their TEM/SEM results mainly due to hydration.

Compared to the Pickering emulsion and surfactant assembly approach used in the synthesis of head-tail

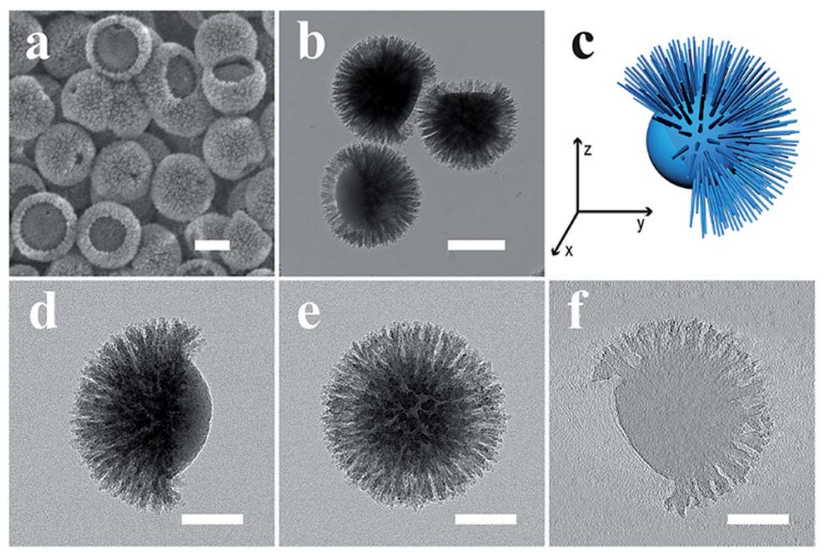

Fig. 2 SEM (a), TEM (b), 3D model (c), tilting TEM ( $d$ and e, from $0^{\circ}$ to $+90^{\circ}$ ) images and the ET slice (f) of J-SNPs. Scale bars are $200 \mathrm{~nm}$ in $a-b$, and $100 \mathrm{~nm}$ in $\mathrm{d}-\mathrm{f}$. asymmetric SNPs, ${ }^{27,28}$ the bottom-up assembly approach developed in this study does not use surfactant templates or preformed seed particles to prepare the ABC type heterotrimeric nanoparticles and J-SNPs. In addition, the J-SNPs have a spiky shell (Fig. 2f), ${ }^{29}$ different from the large-pore tail structure in previous reports. ${ }^{27,28}$

We propose a compromised colloidal interaction model to explain the formation of $\mathbf{A B C}$ type heterotrimeric nanoparticles. In our synthesis, there are two polymerizable systems that can form isolated silica (A) and APF (B) colloidal particles (Fig. 3a) in the absence of EDA. This is because both silica and APF nanoparticles are negatively charged, ${ }^{30}$ and thus there is repulsion or weak interaction between silica and APF, leading to homogenous nucleation and particle growth of both $\mathbf{A}$ and $\mathbf{B}$ particles individually (named as the $\mathbf{A}+\mathbf{B}$ model, see Fig. $3 \mathrm{~b}$ and e). As shown in Fig. 3c, the addition of a high amount of EDA (0.22 $\mathrm{mL}$ ) introduces positive charge on the silica surface and triggers the heterogeneous nucleation and growth of the A@B model (more precisely, $\mathbf{B}$ is the copolymerized silica primary particles and APF, see Fig. 3d and e). Only when the interaction between two colloidal systems is compromised (adjusted by the EDA amount at $0.175 \mathrm{~mL}$ ), not too weak to cause $\mathbf{A}+\mathbf{B}$ growth nor too strong to induce A@B growth, the nucleation of polymerized APF occurs preferentially on one side of the silica core, forming a Janus A-B assembly (see Fig. 3e) (see ESI for detailed discussions $\dagger$ ). The relative size of block $\mathbf{B}$ on each nanoparticle can be easily tuned by the amount of EDA (Fig. S4†).

The reduced amount of EDA could be responsible for the first deposition of the APF block in the Janus A-B assembly model, rather than the APF-silica block at higher EDA amounts in the A@B growth model. With increasing reaction time, the copolymerized SPPs and APF form the third block (C) on the other side of the silica core, forming the ABC type heterotrimeric nanoparticles (Scheme 1a and Fig. 1). This is supported by time-dependent TEM studies of the ABC type heterotrimeric nanoparticles (Fig. 3f-i and f1-i1), which showed that block A was first formed with a silica-dominant composition, followed by the formation of the $\mathbf{A B}$ type Janus structure where the $\mathbf{B}$ block is predominantly APF, and finally the ABC type heterotrimeric nanoparticles with both silica and APF in the $\mathrm{C}$ block (see ESI for detailed discussions $\dagger$ ).

Janus nanoparticles prepared via the bottom-up assembly approach provide novel platforms to investigate the impact of nanoparticle asymmetry on their cellular interactions and drug delivery performance. As a demonstration, the transepithelial transport of Janus nanoparticles was evaluated using an epithelial Caco-2/microfold (M) cell monolayer model. ${ }^{31}$ Caco-2 cells were selected as the model epithelial cell and grown on a porous surface to mimic the gut epithelium. M cells, which are located in the human intestinal epithelium in the gut, are highly specialized for the phagocytosis and transcytosis of gut lumen macromolecules, particulate antigens and microbes across the epithelial barrier. ${ }^{32}$ In the in vitro model, Raji B cells were co-cultured with Caco- 2 cells on transwells to stimulate the differentiation of $\mathrm{M}$ cells (Fig. 4a). ${ }^{31}$ S-SNPs (see Fig. 3d and S5) were chosen as a control sample. The transepithelial transport and cell monolayer internalization were measured separately 


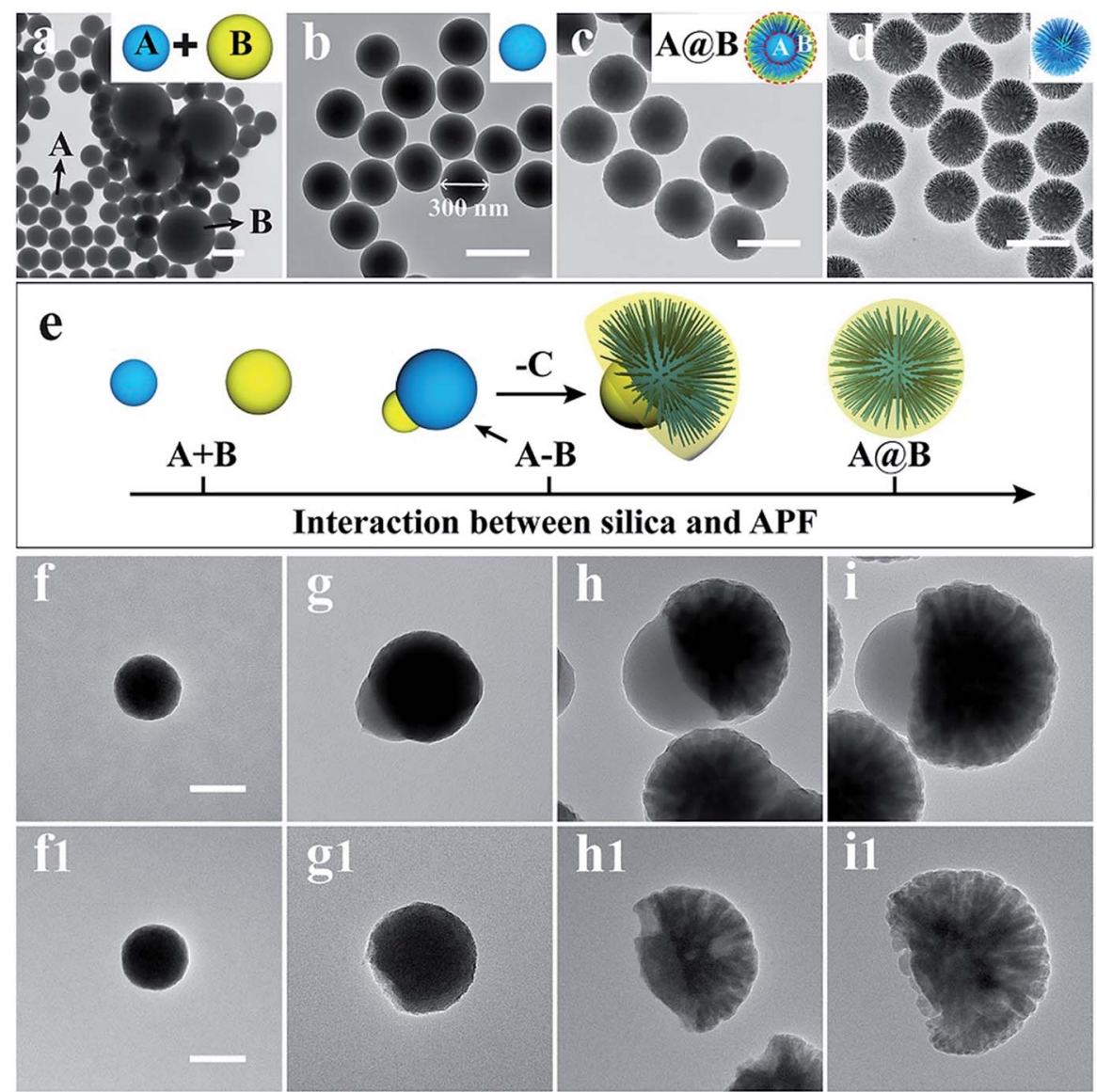

Fig. 3 TEM images of APF-silica nanoparticles with different amounts of EDA. (a and b) $0 \mathrm{~mL}$; (c and d) $0.22 \mathrm{~mL}$. As-synthesized samples (a and $c)$; calcined samples (b and d). Schematic illustration of the three growth models (e). TEM images of morphological evolution of the ABC type heterotrimeric nanoparticle collected at different reaction times. $5 \mathrm{~min}(\mathrm{f}$ and $\mathrm{f} 1$ ); $15 \mathrm{~min}$ ( $\mathrm{g}$ and $\mathrm{g} 1) ; 30 \mathrm{~min}(\mathrm{~h}$ and $\mathrm{h} 1) ; 1 \mathrm{~h}(\mathrm{i}$ and $\mathrm{i} 1)$. The assynthesized composite ( $f-i)$; silica structures ( $f 1-i 1$ ) obtained by calcination of samples in ( $f-i)$. Scale bars are $400 \mathrm{~nm}$ in a-d, and $100 \mathrm{~nm}$ in $f-i$ and $\mathrm{f} 1-\mathrm{i} 1$.

for J-SNPs and S-SNPs (labelled with RITC, Fig. S6 and S7†). As shown in Fig. S8, J-SNPs广 showed higher transported and uptake amounts across the cell monolayer compared with SSNPs, except for short incubation times $(0.5 \mathrm{~h})$, where the uptake amounts were similar. Collectively, the Caco-2/M cell monolayer takes in more J-SNPs than S-SNPs.

Afterwards, a model protein antigen ovalbumin (OVA, conjugated with FITC to give green fluorescence) was loaded into the nanoparticles to further evaluate the protein transport ability of J-SNPs and S-SNPs across the Caco-2/M cell monolayer, which provides useful information for future oral delivery applications. ${ }^{33}$ Characterization of the FITC-OVA loaded SNPs can be found in Fig. S9. $\dagger$ After adding the FITC-OVA loaded NPs (NPs-FITC-OVA) to the apical side of the Caco-2/M cell monolayer, the uptake and transported amounts of the NPs-FITCOVA were evaluated using a plate reader (Fig. 4b) and flow cytometer (Fig. S10 and S11 †). It is found that both the uptake and transported amounts increased with the incubation time $(0.5-4 \mathrm{~h})$, which were determined according to the fluorescence intensity of FITC-OVA in the cell monolayer and the basolateral chamber. The fluorescence intensity was used to reflect the relative uptake and transported amounts of OVA. At $4 \mathrm{~h}$, J-SNPs displayed a much higher transepithelial amount of FITC-OVA than S-SNPs.

To understand the difference in transepithelial transport between J-SNPs and S-SNPs, cells were stained with rhodaminephalloidin to indicate the activation of phagocytosis. It was found that J-SNPs stimulated faster and more intense phagocytosis than S-SNPs (Fig. 4c), suggesting that the higher transcytosis of J-SNPs through the Caco-2/M cell monolayer is associated with their enhanced phagocytosis. From the Z-stack confocal scans (Fig. 4d), the superposition of green (M cells) and red fluorescence (SNPs) suggests that M cells play a main role in transcytosis. At an incubation time of $0.5 \mathrm{~h}$, J-SNPs were observed inside or at the bottom sides of Caco-2/M cells (dashed circle), while S-SNPs were mainly entrapped on the cell surface or on the top side (white arrows) at 0.5 and $2 \mathrm{~h}$, indicating the faster transcytosis of J-SNPs. At a prolonged incubation time of $4 \mathrm{~h}$, both J-SNPs and S-SNPs were found to be located at the bottom side of $\mathrm{M}$ cells, indicating transport through M-cells.

We further compared the protein transport ability of two other conventional spherical silica nanoparticles, dendritic mesoporous silica nanoparticles (DMSNs) and MCM-48 (see Fig. S12 to S15 for more details on characterization $\dagger$ ). FITC-OVA loaded DMSNs and 


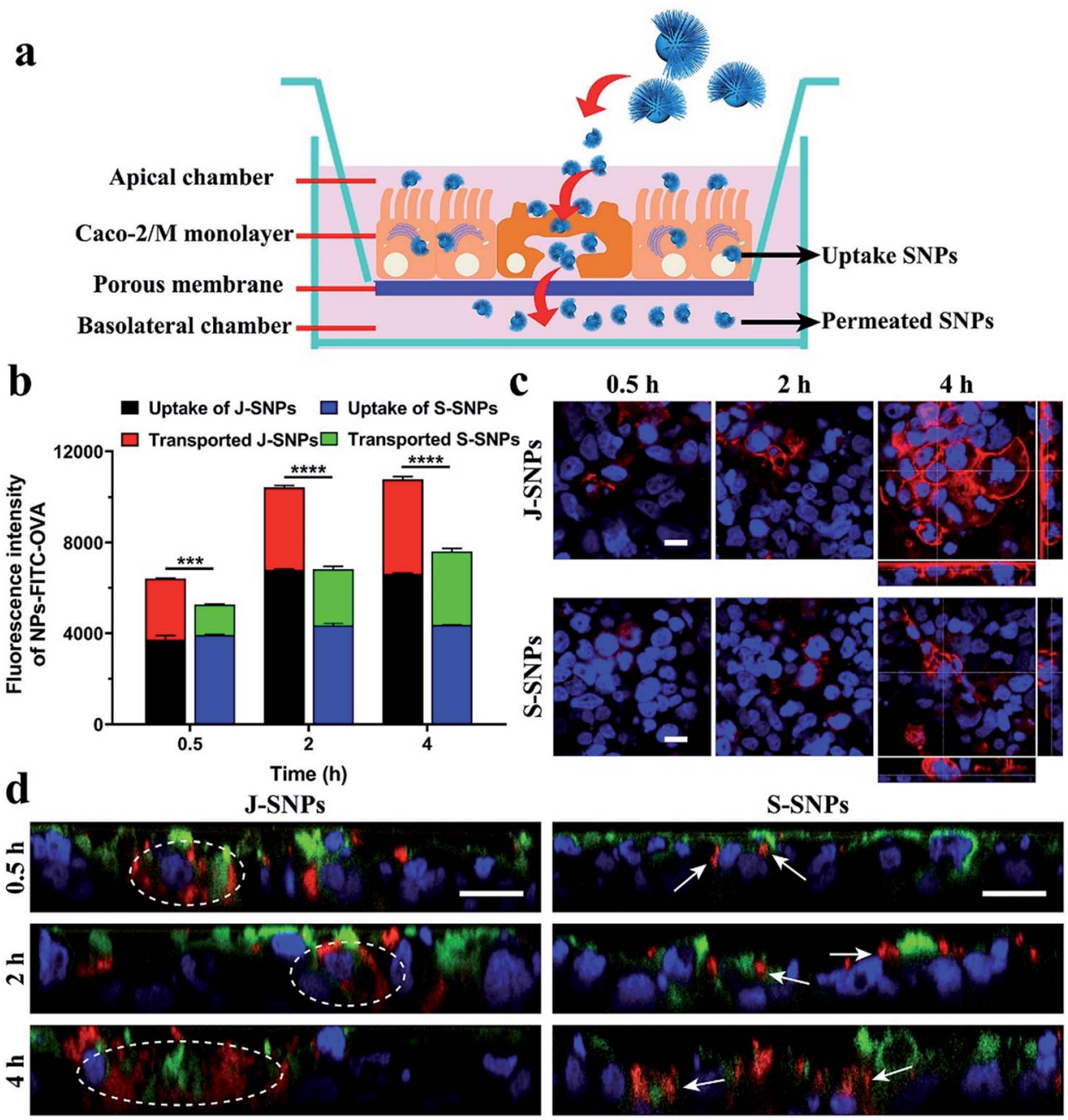

Fig. 4 Schematic illustration of the Caco-2/M-cell monolayer (a). Fluorescence intensity of FITC-OVA loaded J-SNPS and S-SNPs transported through or taken up by the human intestine Caco-2/M-cell monolayer (b). Statistical analyses were performed by using two-way ANOVA. *** indicates $p<0.001, * * * *<<0.0001$. Fluorescence images of the Caco-2/M-cell monolayer treated with J-SNPs and S-SNPs at 0.5, 2 and $4 \mathrm{~h}$ (c). Fluorescence microscopic Z-scans of the Caco-2/M-cell monolayer incubated with J-SNPs and S-SNPs (labelled with RITC, red) for 0.5, 2 and 4 $\mathrm{h}$ (d). Rhodamine phalloidin (red) staining was used in (c). Cell nuclei were stained with Hoechst (blue) in c and d. M cell borders were stained with wheat germ agglutinin Alexa Fluor 488 (green) in (d). All scale bars are $10 \mu \mathrm{m}$.

MCM-48 showed lower uptake and transported amount at low incubation times ( 0.5 and $2 \mathrm{~h}$ ) compared with those of J-SNPs and S-SNPs (Fig. S16 †). At $4 \mathrm{~h}$, the uptake of MCM-48 is similar to that of J-SNPs, however the transported amount is significantly lower than that of J-SNPs. Representative images of the Z-stack confocal scans are shown in Fig. S17, $\uparrow$ the cellular uptake of DMSNs and MCM-48 was found to be increased with the incubation time, while only limited transported nanoparticles were observed, consistent with the results from the protein transepithelial transport experiment. The above results suggest that the asymmetry of nanoparticles plays a significant role in their cellular uptake and transport performance. J-SNPs facilitate faster and enhanced transcytosis across the Caco-2/M cell monolayer than SSNPs, mainly through enhanced phagocytosis by non-professional phagocytic $\mathrm{M}$ cells with high phagocytic capability. ${ }^{34}$

The above conclusion is further supported by cellular uptake and phagocytosis studies of J-SNPs and S-SNPs in four cell lines (Fig. S18-S20†), showing that J-SNPs were internalized more and faster by cells with high phagocytic capability (RAW264.7 and HCT116 cells, Fig. S21-S25†), but not in cells with low phagocytic capability (CHO-K1 and KHOS cells). By inhibiting the phagocytosis pathway in RAW264.7 and HCT116 cells, the uptake of J-SNPs was significantly reduced (Fig. S26†), highlighting the critical role of the phagocytosis pathway in the internalization of J-SNPs.

The reason for the enhanced phagocytosis of J-SNPs over SSNPs in cells with high phagocytic capability is not clear, but is presumably associated with the Janus morphology. SEM observations (Fig. S27 and S28†) revealed that a relatively higher number of J-SNPs appeared "spherical" and partially engulfed in RAW264.7 and HCT116 cells. This trend was not observed in CHO-K1 and KHOS cells. It is suggested that only in cells with high phagocytic capability (e.g., RAW264.7 and HCT116 cells), could the "dent" side contact mode with the cell membrane facilitate phagocytosis, eventually leading to higher cellular uptake of the J-SNPs than S-SNPs. Clearly, this theory should be 
tested in more cell lines and various asymmetric nanoparticles to draw a conclusion.

Finally, the MTT assay of J-SNPs and S-SNPs in the four cell lines (RAW264.7, HCT116, CHO-K1 and KHOS cells) was conducted at different incubation times ( $24 \mathrm{~h}$ and $72 \mathrm{~h}$ ) as shown in Fig. S29 and S30. $\dagger$ Under the particle concentration range of $0-$ $110 \mu \mathrm{g} \mathrm{mL} \mathrm{m}^{-1}$, both J-SNPs and S-SNPs showed dose dependent cytotoxicity in RAW264.7, CHO-K1 and KHOS. RAW264.7 cells were found to be more susceptible compared with other cell lines, probably due to their high phagocytic ability. At $24 \mathrm{~h}$ of incubation in RAW264.7, J-SNPs showed a higher cytotoxicity compared to S-SNPs. At prolonged time points, both J-SNPs and S-SNPs showed similar dose dependent cytotoxicity regardless of the shape of the nanoparticles, consistent with literature reports. HCT116 cells showed higher cell viability than other cell lines and no significant dose dependent behaviour was observed, indicating that HCT116 cancer cells were more resistant to SNPs under the experimental conditions. ${ }^{35,36}$

\section{Experimental}

\section{Synthesis of the ABC type heterotrimeric nanoparticles}

Monodisperse ABC type heterotrimeric nanoparticles were synthesized through a one-pot surfactant free process under alkaline conditions in an alcohol-water system. Typically, 3aminophenol $(0.41 \mathrm{~g})$, formalin $(37 \mathrm{wt} \%, 0.9 \mathrm{~mL})$, and tetraethyl orthosilicate (TEOS, $1.75 \mathrm{~mL}$ ) were added to the solution composed of ammonia aqueous solution ( $28 \mathrm{wt} \%, 1.56 \mathrm{~mL}$ ), deionized water $(10 \mathrm{~mL})$, ethylenediamine (EDA, $0.175 \mathrm{~mL})$ and ethanol $(40 \mathrm{~mL})$. The above solution was vigorously stirred at room temperature for $4 \mathrm{~h}$. The as-synthesized particles were separated by centrifugation, and washed with ethanol and deionized water three times. The final product was obtained by drying at $50{ }^{\circ} \mathrm{C}$ overnight. The J-SNPs were obtained by calcination of the pre-dried $\mathbf{A B C}$ type heterotrimeric nanoparticles at $550{ }^{\circ} \mathrm{C}$ in air.

\section{Conclusions}

In summary, a bottom-up surfactant-free self-assembly route is developed for the synthesis of $\mathbf{A B C}$ type heterotrimeric nanoparticles and Janus-type silica nanoparticles. This finding leads to a variety of nanoparticles with controllable morphologies and compositions, which will be useful tools in future studies to understand the nanoparticle-cell interactions. Moreover, JSNPs have enhanced transport through an in vitro epithelial monolayer, indicating the potential application of J-SNPs as oral delivery vectors with improved delivery efficiency.

\section{Conflicts of interest}

There are no conflicts to declare.

\section{Acknowledgements}

The authors acknowledge the support from the Australian Research Council, Australian Microscopy and Microanalysis
Research Facility at the Centre for Microscopy and Microanalysis (CMM), University of Queensland, and the Queensland node of the Australian National Fabrication Facility. J. Y. Fu acknowledges the award of Chinese Scholarship Council Scholarship.

\section{Notes and references}

1 A. Walther and A. H. Müller, Janus particles, Soft Matter, 2008, 4, 663.

2 A. Walther and A. H. Müller, Janus particles: synthesis, selfassembly, physical properties, and applications, Chem. Rev., 2013, 113, 5194

3 J. Chen, S. M. Andler, J. M. Goddard, S. R. Nugen and V. M. Rotello, Integrating recognition elements with nanomaterials for bacteria sensing, Chem. Soc. Rev., 2017, 46, 1272.

4 S. Mitragotri and J. Lahann, Physical approaches to biomaterial design, Nat. Mater., 2009, 8, 15.

5 G. De Crozals, R. Bonnet, C. Farre and C. Chaix, Nanoparticles with multiple properties for biomedical applications: a strategic guide, Nano Today, 2016, 11, 435.

$6 \mathrm{~J}$. Hu, S. Zhou, Y. Sun, X. Fang and L. Wu, Fabrication, properties and applications of Janus particles, Chem. Soc. Rev., 2012, 41, 4356.

7 J. L. Fenton, B. C. Steimle and R. E. Schaak, Tunable intraparticle frameworks for creating complex heterostructured nanoparticle libraries, Science, 2018, 360, 513.

8 P. C. Chen, X. Liu, J. L. Hedrick, Z. Xie, S. Wang, Q. Y. Lin, M. C. Hersam, V. P. Dravid and C. A. Mirkin, Polyelemental nanoparticle libraries, Science, 2016, 352, 1565.

9 A. G. Mark, J. G. Gibbs, T. C. Lee and P. Fischer, Hybrid nanocolloids with programmed three-dimensional shape and material composition, Nat. Mater., 2013, 12, 802.

10 J. M. Hodges, J. R. Morse, M. E. Williams and R. E. Schaak, Microscopic investigation of chemoselectivity in Ag-Pt$\mathrm{Fe}_{3} \mathrm{O}_{4}$ heterotrimer formation: mechanistic insights and implications for controlling high-order hybrid nanoparticle morphology, J. Am. Chem. Soc., 2015, 137, 15493.

11 C. G. Read, T. R. Gordon, J. M. Hodges and R. E. Schaak, Colloidal hybrid nanoparticle insertion reaction for transforming heterodimers into heterotrimers, J. Am. Chem. Soc., 2015, 137, 12514.

12 B. Y. Guan, L. Yu and X. W. Lou, Formation of asymmetric bowl-like mesoporous particles via emulsion-induced interface anisotropic assembly, J. Am. Chem. Soc., 2016, 138, 11306.

13 W. Qin, T. Peng, Y. Gao, F. Wang, X. Hu, K. Wang, J. Shi, D. Li, J. Ren and C. Fan, Catalysis-driven selfthermophoresis of Janus plasmonic nanomotors, Angew. Chem., Int. Ed., 2017, 56, 515.

14 D. Yi, Q. Zhang, Y. Liu, J. Song, Y. Tang, F. Caruso and Y. Wang, Synthesis of chemically asymmetric silica nanobottles and their application for cargo loading and as nanoreactors and nanomotors, Angew. Chem., Int. Ed., 2016, 55, 14733. 
15 X. M. Li, L. Zhou, Y. Wei, A. M. El-Toni, F. Zhang and D. Y. Zhao, Anisotropic growth-induced synthesis of dualcompartment Janus mesoporous silica nanoparticles for bimodal triggered drugs delivery, J. Am. Chem. Soc., 2014, 136, 15086.

16 X. M. Li, L. Zhou, Y. Wei, A. M. El-Toni, F. Zhang and D. Y. Zhao, Anisotropic encapsulation-induced synthesis of asymmetric single-hole mesoporous nanocages, J. Am. Chem. Soc., 2015, 137, 5903.

17 F. Wang, G. M. Pauletti, J. T. Wang, J. M. Zhang, R. C. Ewing, Y. L. Wang and D. L. Shi, Dual Surface-functionalized Janus nanocomposites of polystyrene $/ \mathrm{Fe}_{3} \mathrm{O}_{4} @ \mathrm{SiO}_{2}$ for simultaneous tumor cell targeting and stimulus-induced drug release, Adv. Mater., 2013, 25, 3485.

18 G. Y. Chen, I. Roy, C. H. Yang and P. N. Prasad, Nanochemistry and nanomedicine for nanoparticle-based diagnostics and therapy, Chem. Rev., 2016, 116, 2826.

19 S. Wilhelm, A. J. Tavares, Q. Dai, S. Ohta, J. Audet, H. F. Dvorak and W. C. Chan, Analysis of nanoparticle delivery to tumours, Nat. Rev. Mater., 2016, 1, 16014.

20 T. L. Moore, L. Rodriguez-Lorenzo, V. Hirsch, S. Balog, D. Urban, C. Jud, B. Rothen-Rutishauser, M. Lattuada and A. Petri-Fink, Nanoparticle colloidal stability in cell culture media and impact on cellular interactions, Chem. Soc. Rev., 2015, 44, 6287.

21 H. Yang, Z. Chen, L. Zhang, W. Y. Yung, K. C. F. Leung, H. Y. E. Chan and C. H. J. Choi, Mechanism for the cellular uptake of targeted gold nanorods of defined aspect ratios, Small, 2016, 12, 5178.

22 Y. Li, M. Kröger and W. K. Liu, Shape effect in cellular uptake of PEGylated nanoparticles: comparison between sphere, rod, cube and disk, Nanoscale, 2015, 7, 16631.

$23 \mathrm{~K}$. Yang and Y. Q. Ma, Computer simulation of the translocation of nanoparticles with different shapes across a lipid bilayer, Nat. Nanotech., 2010, 5, 579.

24 C. C. Carcouët, M. W. Van De Put, B. Mezari, P. C. Magusin, J. Laven, P. H. Bomans, H. Friedrich, A. C. C. Esteves, N. A. Sommerdijk, R. A. Van Benthem and G. de With, Nucleation and growth of monodisperse silica nanoparticles, Nano Lett., 2014, 14, 1433.

25 W. Stöber, A. Fink and E. Bohn, Controlled growth of monodisperse silica spheres in the micron size range, $J$. Colloid Interface Sci., 1968, 26, 62.
26 H. Friedrich, P. E. de Jongh, A. J. Verkleij and K. P. de Jong, Electron tomography for heterogeneous catalysts and related nanostructured materials, Chem. Rev., 2009, 109, 1613.

27 P. L. Abbaraju, A. K. Meka, H. Song, Y. Y. Yang, M. Jambhrunkar, J. Zhang, C. Xu, M. H. Yu and C. Z. Yu, Asymmetric silica nanoparticles with tunable head-tail structures enhance hemocompatibility and maturation of immune cells, J. Am. Chem. Soc., 2017, 139, 6321.

28 P. L. Abbaraju, M. Jambhrunkar, Y. Y. Yang, Y. Liu, Y. Lu and C. Z. Yu, Asymmetric mesoporous silica nanoparticles as potent and safe immunoadjuvants provoke high immune responses, Chem. Commun., 2018, 54, 2020.

29 H. Song, Y. Ahmad Nor, M. H. Yu, Y. N. Yang, J. Zhang, H. W. Zhang, C. Xu, N. Mitter and C. Z. Yu, Silica nanopollens enhance adhesion for long-term bacterial inhibition, J. Am. Chem. Soc., 2016, 138, 6455.

30 N. Li, Q. Zhang, J. Liu, J. Joo, A. Lee, Y. Gan and Y. D. Yin, Sol-gel coating of inorganic nanostructures with resorcinol-formaldehyde resin, Chem. Commun., 2013, 49, 5135.

31 C. Schimpel, B. Teubl, M. Absenger, C. Meindl, E. Fröhlich, G. Leitinger, A. Zimmer and E. Roblegg, Development of an advanced intestinal in vitro triple culture permeability model to study transport of nanoparticles, Mol. Pharm., 2014, 11, 808.

$32 \mathrm{H}$. Cheroutre and L. Madakamutil, Acquired and natural memory $\mathrm{T}$ cells join forces at the mucosal front line, Nat. Rev. Immunol., 2004, 4, 290.

33 M. R. Neutra, N. J. Mantis and J. P. Kraehenbuhl, Collaboration of epithelial cells with organized mucosal lymphoid tissues, Nat. Immunol., 2001, 2, 1004.

34 Phagocytosis by nonprofessional phagocytes, in Advances in cellular and molecular biology of membranes and organelles, ed. S. Gordon, JAI Press, Stamford, CT, 1999.

35 T. Yu, A. Malugin and H. Ghandehari, Impact of silica nanoparticle design on cellular toxicity and hemolytic activity, ACS Nano, 2011, 5, 5717.

36 J. S. Chang, K. L. B. Chang, D. F. Hwang and Z. L. Kong, In vitro cytotoxicity of silica nanoparticles at high concentrations strongly depends on the metabolic activity type of the cell line, Environ. Sci. Technol., 2007, 41, 2064. 